\title{
Comparison of Reconstruction and Control algorithms on the ESO end-to-end simulator OCTOPUS
}

\author{
I. Montilla ${ }^{1, a}$, C. Béchet ${ }^{2}$, M. LeLouarn ${ }^{2}$, C. Correia $^{3}$, M. Tallon ${ }^{4}$, M. Reyes ${ }^{1}$, and É. Thiébaut ${ }^{4}$ \\ 1 Instituto de Astrofísica de Canarias, Santa Cruz de Tenerife 38205, Spain \\ 2 European Southern Observatory, Garching bei München 85748, Germany \\ 3 Office National d'Etudes et de Recherches Aérospatiales, 29 Av. de la Division Leclerc, 92322 \\ Chatillon, France \\ 4 Université de Lyon, Lyon, F-69003, France ; Université Lyon 1, Observatoire de Lyon, 9 avenue \\ Charles André, Saint-Genis Laval, F-69230, France ; CNRS, UMR 5574, Centre de Recherche As- \\ trophysique de Lyon; Ecole Normale Supérieure de Lyon, Lyon, F-69007, France
}

\begin{abstract}
Extremely Large Telescopes are very challenging concerning their Adaptive Optics requirements. Their diameters, the specifications demanded by the science for which they are being designed for, and the planned use of Extreme Adaptive Optics systems, imply a huge increment in the number of degrees of freedom in the deformable mirrors. It is necessary to study new reconstruction algorithms to implement the real time control in Adaptive Optics at the required speed. We have studied the performance, applied to the case of the European ELT, of three different algorithms: the matrix-vector multiplication (MVM) algorithm, considered as a reference; the Fractal Iterative Method (FrIM); and the Fourier Transform Reconstructor (FTR). The algorithms have been tested on ESO's OCTOPUS software, which simulates the atmosphere, the deformable mirror, the sensor and the closed-loop control. The MVM is the default reconstruction and control method implemented in OCTOPUS, but it scales in $O\left(N^{2}\right)$ operations per loop so it is not considered as a fast algorithm for wave-front reconstruction and control on an Extremely Large Telescope. The two other methods are the fast algorithms studied in the E-ELT Design Study. The performance, as well as their response in the presence of noise and with various atmospheric conditions, has been compared using a Single Conjugate Adaptive Optics configuration for a $42 \mathrm{~m}$ diameter ELT, with a total amount of 5402 actuators. Those comparisons made on a common simulator allow to enhance the pros and cons of the various methods, and give us a better understanding of the type of reconstruction algorithm that an ELT demands.
\end{abstract}

\section{Introduction}

We are in the era of the Extremely Large Telescopes (ELT). Many of these are being designed all over the world, the European Extremely Large Telescope (E-ELT) among them. The European-ELT is a project jointly funded by the European Southern Observatory (ESO) and the European Community. The preliminary design of the facility started in 2007, and the goal is to have the E-ELT Observatory operational around 2015. A large group of European Institutes are developing critical studies and technologies to make it possible. The latest proposed design consists of a telescope with a diameter of up to $42 \mathrm{~m}$, and an f-ratio of 12. The E-ELT scientific goals have been described by Hook et al. [1], and among the most important scientific cases we can find the detection and characterization of earthlike planets in extrasolar systems, the study of protoplanetary disks and galaxy formation, observing high-redshift galaxies and studying the re-ionization of the early universe. All of them require not only a large collecting area, but also a good spatial resolution. It is essential to build any ELT with a powerful Adaptive Optics (AO) system to unleash all its scientific potential. For example, for the detection of exo-earth planets, a high Strehl ratio of 70\%-90\% will be required, and to study the inner region of circumstellar disks, diffraction-limited resolution is needed. The computational burden to obtain such correction at the required speed is too large for the classical AO algorithms, demanding faster high-performance algorithms to be developed.

\footnotetext{
a e-mail: imontilla@iac.es
}

This is an Open Access article distributed under the terms of the Creative Commons Attribution-Noncommercial License, which permits unrestricted use, distribution, and reproduction in any noncommercial medium, provided the original work is properly cited. 


\section{Description of the methods}

\subsection{Matrix-Vector Multiply}

The Matrix-Vector Multiply is the standard reconstruction method (see Hunt [2]). The wavefront $\phi$ is related to the phase difference vector $\mathbf{s}$ through the matrix $\mathbf{P}$ :

$$
\begin{gathered}
\mathbf{s}=\mathbf{P} \cdot \phi+\mathbf{n}, \\
\hat{\phi}=\mathbf{R} \cdot \mathbf{s},
\end{gathered}
$$

where $\mathbf{R}$ is the linear estimator computed to calculate the reconstructed wavefront, usually by an inversion. The method scales in $O\left(N^{2}\right)$ operations per loop so it is not considered as a "fast algorithm" for wave-front reconstruction and control on an Extremely Large Telescope, but we have used it as a reference to compare the results of the other reconstructors.

\subsection{Fourier Transform Reconstructor}

The wavefront reconstruction method using a Discrete Fourier Transform was first proposed by Freischlad [3], and later adapted by Poyneer [4] for use on the Fried geometry. It basically consists in reconstructing the wavefront in Fourier space, by applying a certain filter to the Fourier transform of the gradients of the subapertures sampled by the detector. The gradients provided by the detector are $s_{x}[m, n]$ and $s_{y}[m, n]$, that in the case of the Shack-Hartmann geometry are:

$$
\begin{aligned}
& s_{x}[m, n]=1 / 2(\phi[m, n+1]-\phi[m, n]+\phi[m+1, n+1]-\phi[m+1, n]), \\
& s_{y}[m, n]=1 / 2(\phi[m+1, n]-\phi[m, n]+\phi[m+1, n+1]-\phi[m, n+1]) .
\end{aligned}
$$

Their Fourier transforms are $S_{x}[k, l]$ and $S_{y}[k, l]$. The reconstructed wavefront in the Fourier space is:

$$
\widehat{\phi}[k, l]= \begin{cases}0, & \text { if } k, l=0, N / 2 \\ F_{x}[k, l] \cdot S_{x}[k, l]+F_{y}[k, l] \cdot S_{y}[k, l], & \text { else }\end{cases}
$$

where $F_{x}[k, l]$ and $F_{y}[k, l]$ are the coefficients of the filter derived for a given geometry. In our case, we use the modified-Hudgin filter. In a real system, gradients are only available typically on a circular aperture, and the measurements have to be extended to a rectangular grid and some periodicity has to be imposed to the extended data. We are not going to focus on these details, for more information on extension methods, as well as for the details on the filters, we refer to Poyneer [4]. The method scales in $O(N \log N)$ operations per loop.

\subsection{Fractal Iterative Method}

FrIM is a minimum variance reconstructor (see Bechet et al. [5]). It takes advantage of the fractal structure of the atmospheric turbulence to define a change of variables:

$$
\hat{\phi}=\mathbf{K} \cdot \hat{\mathbf{u}}
$$

where $\hat{\mathbf{u}}$ are the coefficients of statistically independent modes on the phase map and $\mathbf{K}$ an invertible fractal operator. The system to solve iteratively is then:

$$
\mathbf{A} \cdot \hat{\mathbf{u}}=\mathbf{b},
$$

with $\mathbf{A}, \mathbf{b}$ being computed from $\mathbf{s}, \mathbf{P}, \mathbf{K}$. The method scales in $O(N)$ operations per loop. 


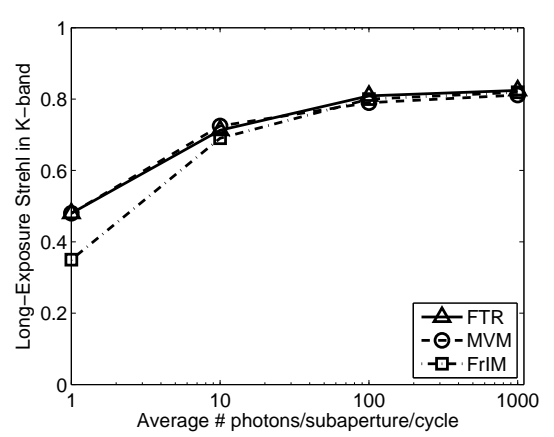

Fig. 1. Long-exposure strehl vs. magnitude

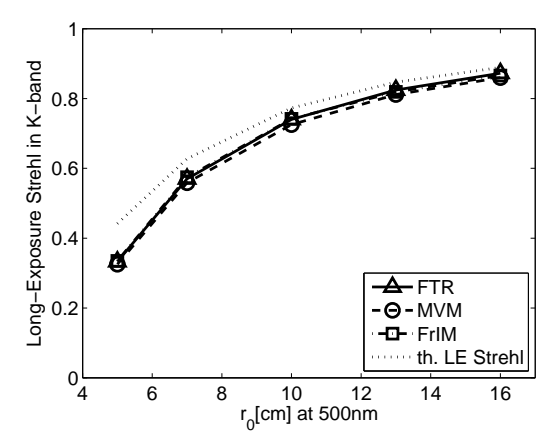

Fig. 2. Long-exposure strehl vs. $r_{0}$

\section{Summary of performance results}

The tests that we present in this document have been done with the first light Single Conjugate Adaptive Optics configuration for the E-ELT. This means a telescope diameter of $42 \mathrm{~m}$, with a central obscuration of $28 \%$ of the diameter. The pupil is circular, and segmentation co-phasing errors are neglected. Octopus simulates this telescope altogether with the adaptive optics system. The wave-front sensor (WFS) is a Shack-Hartmann and a squared $84 \times 84$-subaperture array is assumed. This gives a sub-aperture size on the sky of $50 \mathrm{~cm}$. The Deformable Mirror (DM) is simulated with bilinear influence functions. It is a circular mirror with a regular sampling of square geometry and 85 actuators across the diameter. The total amount of actuators is 5402. The DM is used in Fried's geometry with respect to the WFS. This means that the actuators are located at positions conjugated to the corners of the sub-apertures. Some parameters of the system have been kept fixed for all the tests: the frame rate is $1 \mathrm{kHz}$ (for phase screens displacement evaluation), the correction delay is 2 frames, there is no RON but the photon noise is simulated.

\subsection{Performance vs. star magnitude}

First, we have tested the dependency of the performance on the star magnitude, setting the following configuration: $\tau_{0}=3 \mathrm{~ms}$ and $\mathrm{r}_{0}=0.13 \mathrm{~m}$. Tests were performed for 1000, 100, 10, and 1 ph.subaperture ${ }^{-1}$. cycle $^{-1}$. The curves are plotted in Figure 1, comparing the long-exposure Strehl evolution for the 3 methods. For each case, FTR and MVM methods have optimized the gain of the integrator. On the contrary, FrIM hyperparameter has been maintained constant at 0.1 . For a magnitude of 1000 and 100 ph.subaperture ${ }^{-1}$.cycle ${ }^{-1}$ the Strehl is higher than $80 \%$ for the three algorithms. A slight decrease (70\%) appears for the 10-photon case, but all methods seem to behave identically. For these three values of magnitude, it is the reconstruction performance of the algorithms that is characterized, more than the control strategy. The reached Strehl is limited by constant fitting and delay errors and by wave-front reconstruction error, which is minor in the 1000- and 100-photon cases and which is slightly higher for the 10-photon case. The measurement uncertainties are larger when only 10 photons are received on average by sub-aperture, providing worse reconstruction and correction. As a consequence, this shows that all three methods include good reconstructors, even for large noise on the data ( $c f .10$-photon case).

\subsection{Performance vs. turbulence conditions}

Following, we performed some tests to determine the behavior of the different methods with respect to turbulence conditions. The changes in the turbulence are illustrated by two different studies: one depending on Fried's parameter value, $r_{0}$, and another depending on the coherence time value, $\tau_{0}$. With high flux conditions, the reachable Strehl is mainly driven by the sum of two error contributions: the 
fitting error and the delay error. The first one is characterized by the ratio between the actuators pitch and Fried's parameter, according to Hudgin's formula:

$$
\sigma_{\text {fitting }}^{2}=0.287\left(d / r_{0}\right)^{5 / 3}
$$

The second one, in case no temporal prediction is made, can be estimated using the following expression:

$$
\sigma_{\text {delay }}^{2}=6.88\left(V \tau / \tau_{0}\right)^{5 / 3}
$$

On the one hand, the tests of section 3.2.1 investigate the reachable Strehl when the fitting error $\sigma_{\text {fitting }}^{2}$ becomes dominant in the budget error. On the other hand, the tests of section 3.2.2 concentrate on the cases when $\sigma_{\text {delay }}^{2}$ may dominate. For all the simulated conditions, the theoretical sum of these two contributions to the error is represented as an equivalent reachable Strehl value. Since the two contributions are considered to be uncorrelated, this value is an approximation and can be considered as a qualitative orientation to know if the methods are into the estimated error budget.

\subsubsection{Fried's parameter}

We tested different values of $r_{0}$, to compare the level of turbulence the various algorithms were able to correct. The configuration has the fixed following characteristics: $\tau_{0}=3 \mathrm{~ms}$ and $1000 \mathrm{ph}$.subaperture ${ }^{-1}$. cycle ${ }^{-1}$. For an $\mathrm{r}_{0}$ of $16 \mathrm{~cm}$, the K-band Strehl is $87 \%$, while it decreases to around $70 \%$ for an $\mathrm{r}_{0}$ of $10 \mathrm{~cm}$. For $\mathrm{r}_{0}=7 \mathrm{~cm}$ the Strehl is $55 \%$. For a very bad case, an $\mathrm{r}_{0}$ of $5 \mathrm{~cm}$, the K-band Strehl still converges but it is a bit lower than 35\%. All the curves are plotted in Figure 2. All the methods show approximately the same performance on these various cases, demonstrating that they provide equivalent reconstruction quality. Furthermore, this reconstruction quality is in agreement with the predicted performance due to the limiting fitting error.

\subsubsection{Coherence time}

We did tests to check the behavior of the performance for different coherence times. The configuration has the fixed following characteristics: $\mathrm{r}_{0}=0.13 \mathrm{~m}$ and $1000 \mathrm{ph}$.subaperture ${ }^{-1}$.cycle ${ }^{-1}$. One can observe from these results that for a coherence time larger than $1 \mathrm{~ms}$ the performance is limited by the number of actuators, for the three algorithms. In other words, the fitting error is dominating; therefore a larger coherence time does not significantly improve the performance. For shorter coherence times, a slight degradation of the performance occurs for all the methods. The lower limit corresponds to $1 \mathrm{~ms}$, with the Strehl decreasing down below $80 \%$ (see Figure 3). These tests also confirm that the reconstruction quality is good and that the temporal delay in such $\mathrm{AO}$ configuration is not a big issue, as soon as the fitting error is already a major limit for the AO system performance.

\subsection{Actuators shift with respect to sub-apertures corners}

Finally, we have performed simulations for different actuators displacements to test how robust the three methods are regarding mis-registrations in the positions of the DM actuators (see Figure 4). The goal is to test what happens to the performance when the actuators are not placed where they should be. Each sub-aperture is simulated with 16 pixels (arbitrary unit) along its side, and we shifted the position of the actuators by 2, 4, 6 and 8 of these pixels respectively, without giving the information to the algorithms. This aims at testing the control algorithm, with respect to modeling errors or calibration errors. A general comment is that the MVM method seems to be more affected by these modeling errors. An unstable behavior is observed for a shift of one third of a sub-aperture. On the contrary, for the two other algorithms the response is not affected for such a mis-registration. More precisely, a longer simulation (of 2000 loops) with FrIM and the FTR method has demonstrated that their control is really stable for a shift of 6 pixels, with a constant Strehl of about 75\%. However, the 8-pixel shift case tested on 2000 loops for those two methods led to instability, driving the Strehl progressively to $0 \%$. 


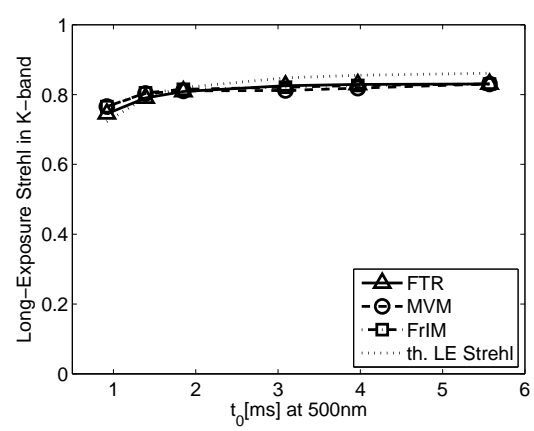

Fig. 3. Long exposure-strehl vs. $t_{0}$

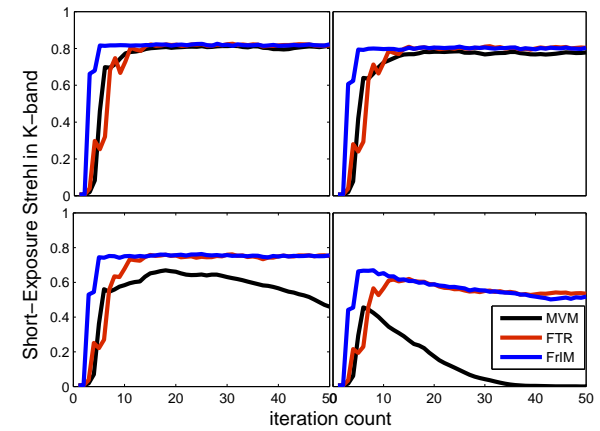

Fig. 4. Comparison of Short-Exposure and LongExposure strehl vs. iteration number as a function of the actuatorś position misregistrations

\section{Time analysis}

We have compared the number of operations for the MVM, the FTR and FrIM SCAO control algorithms. Along with the performance, the speed of an algorithm is one of the key issues to decide which one to use depending on the number of sub-apertures or actuators. The number of operations required to perform the reconstruction gives us an idea of how fast, how complex, or how expensive an algorithm is to be used. With the MVM method we have to multiply the control matrix $\pi \cdot\left(N_{\text {sub }} / 2\right)^{2} \times 2\left(\pi \cdot\left(N_{\text {sub }} / 2\right)^{2}-N_{\text {sub }}\right)$ by the centroids vector $2\left(\pi \cdot\left(N_{\text {sub }} / 2\right)^{2}-N_{\text {sub }}\right) \times 1$. The number of operations for the reconstruction to be performed is, approximately:

$$
N_{o p}^{M V M}=4\left(\pi^{2}\left(N_{\text {sub }} / 2\right)^{4}-\pi N_{\text {sub }}^{3} / 4\right)-\pi\left(N_{\text {sub }} / 2\right)^{2},
$$

where $N_{s u b}$ is the number of subapertures across the aperture. According to [4], the number of operations with the FTR method using the Hudgin filter is:

$$
N_{o p}^{F T R}=2 \cdot N_{s u b} \cdot\left(N-\frac{\pi N_{s u b}}{4}\right)+4 \cdot N^{2}+9 \cdot N^{2} \cdot \log _{2}(N) .
$$

where $N_{\text {sub }}$ is the number of subapertures across the aperture, and $N$ is a power of 2 . The number of subpaertures varies from $N$ to $N / 2$. The worst case is to have small apertures in large grids. FrIM is an iterative method, so that the number of operations to control a SCAO system with this algorithm highly depends on the number of iterations applied per loop. The number of operations for the method is:

$$
N_{o p}^{F r I M}=\left(26+34 \times n_{i t e r}\right) \cdot N^{2},
$$

being $N$ of the form $2^{p}+1$, with $p$ an integer, and $N$ larger than the number of actuators across the aperture. The first tests on Octopus simulator were done with at least $n_{\text {iter }}=5$ iterations per loop, in order to easily analyze the performance of the method with respect to reconstruction quality on one side and control issues on another side. The tests presented here were done with $n_{\text {iter }}=5$ also. However, FrIM has been widely tested on Octopus now and it has been shown to provide optimal performance with only 2 iterations. In Figure 5 we have plotted the number of operations as a function of the number of subapertures across the aperture, for the three algorithms, highlighting three configurations: the one for SPHERE, with 40x40 DoF's (see [6]), for EPICS, with 210x210 (see [7]), and the SCAO configuration for the E-ELT, with $84 \times 84$. For the latter, the particular configuration we have simulated in this report, the MVM algorithm implies about $1.09 \times 10^{8}$ operations, while FrIM with the internal model control and $n_{\text {iter }}=2$ requires around $1.5 \times 10^{6}$ operations and the FTR around $1.1 \times 10^{6}$. This means that FrIM and FTR methods require respectively 70 times and 100 times fewer operations than the MVM method in this configuration. 


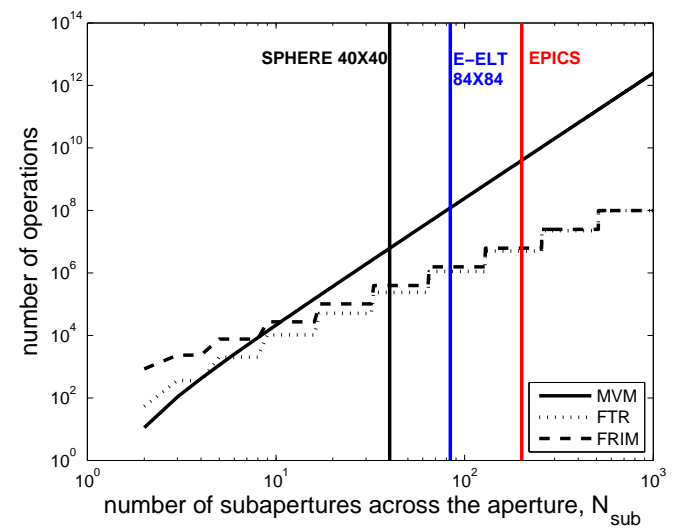

Fig. 5. Number of operations vs. subapertures for the three algorithms on study

\section{Conclusions}

The three algorithms have a very good performance and a robust response. At faint magnitudes, MVM and FTR reach $50 \%$ strehl and FrIM 35\%. We attribute this to the temporal control inside FrIM, not to its reconstructing capabilities. This issue is being studied by C. Béchet. FrIM and FTR seem to be insensitive to modelling errors even for a shift of one third of a subaperture, while the MVM is unstable in such conditions. This is important to relax the specifications on the calibration procedure of the AO system. FrIM and FTR require a fewer number of operations to perform the reconstruction. A subject to be further discussed is which type of parallelization and hardware fits better each algorithm. This issue has been previously addressed (see Montilla et al. [8]) but it has to be studied in depth to determine how the latency can be minimized and in which extent.

\section{Acknowledgments}

This project forms part of the ELT Design Study and is supported by the European Commission, within Framework Programme 6.

\section{References}

1. I.M. Hook, ESO report (2005)

2. B.R. Hunt, Journal of the Optical Society of America A 69 (1979)

3. K.R. Freischlad, C.L. Koliopoulos, Journal of the Optical Society of America A 3 (1986)

4. L. Poyneer, D. Gavel, J. Brase, Journal of the Optical Society of America A 19 (2002)

5. C. Béchet, M. Tallon, E. Thiébaut, FRIM: minimum-variance reconstructor with a FRactal Iterative Method, in Advances in Adaptive Optics (2006), Vol. 6272 of SPIE Conference Series

6. J.L. Beuzit, M. Feldt, K. Dohlen, D. Mouillet, P. Puget, F. Wildi, L. Abe, J. Antichi, A. Baruffolo, P. Baudoz et al., SPHERE: a planet finder instrument for the VLT, in Ground-based and Airborne Instrumentation for Astronomy II (2008), Vol. 7014 of SPIE Conference Series

7. M.E. Kasper, J.L. Beuzit, C. Verinaud, N. Yaitskova, P. Baudoz, A. Boccaletti, R.G. Gratton, N. Hubin, F. Kerber, R. Roelfsema et al., EPICS: the exoplanet imager for the E-ELT, in Adaptive Optics Systems (2008), Vol. 7015 of SPIE Conference Series

8. I. Montilla, M. Reyes, M. Le Louarn, J.G. Marichal-Hernández, J.M. Rodríguez-Ramos, L.F. Rodríguez-Ramos, Performance of the Fourier transform reconstructor for the European Extremely Large Telescope, in Adaptive Optics Systems (2008), Vol. 7015 of SPIE Conference Series 